\title{
Decay detection of constructional softwoods using machine olfaction
}

\author{
Masaki Suzuki ${ }^{*}{ }^{*}$, Teruhisa Miyauchi ${ }^{1}$, Shinichi Isaji ${ }^{1}$, Yasushi Hirabayashi ${ }^{1}$ and Ryuichi Naganawa ${ }^{2}$
}

\begin{abstract}
Fungal decomposition of wood severely affects the soundness of timber constructions. The diagnosis of wood decay requires direct observations or sampling by skilled experts. Wood decay often occurs in obscure spaces, including the enclosed inner spaces of walls or under the floor. In this study, we examined the ability of machine olfaction to detect odors of fungi grown on common construction softwoods to provide a novel diagnostic method for wood construction soundness. The combination of a simple device equipped with semiconductor gas sensors (gas sensor array) and multivariate analysis discriminated a fungi-related odor from control odor without instrumental analysis (e.g., gas chromatography). This method is often referred to as machine olfaction or electronic nose. We measured the odor of wood test pieces that were infected with Fomitopsis palustris or Trametes versicolor and sound test pieces using a gas sensor array. The sensor responses of the specimens showed different patterns between the inoculated and control samples. Each specimen class formed independent groups in a principal component score plot, almost regardless of wood species, fungal species, or cultivation period. This method provides a new decay diagnosis method that is costeffective and easy to operate.
\end{abstract}

Keywords: Wood decay, Wood-decay fungi, Gas sensors, Machine olfaction, Electronic nose

\section{Introduction}

The biological decomposition of wood severely affects the soundness of timber structures, and fungal degradation is the most common cause. Diagnosis of wood decay requires direct observation and/or destructive sampling. Therefore, in narrowly enclosed spaces, including the internal spaces of walls, wood decay detection is difficult. The detection of airborne substances such as microbial volatile organic compounds (MVOCs) or their odor from wood-decay fungi has been studied to overcome this difficulty. Kauhanen et al. [1] demonstrated that trained dogs can detect wood-decay fungi. Konuma et al. [2,3] reported the detailed chemical composition of MVOCs emitted by Fomitopsis palustris (brown-rot) and Trametes versicolor (white-rot) using several instrumental analysis methods. For termite detection, another

\footnotetext{
*Correspondence: suzuki-masaki@hro.or.jp

${ }^{1}$ Forest Products Research Institute, Hokkaido Research Organization,

1-10 Nishikagura, Asahikawa, Hokkaido 071-0198, Japan

Full list of author information is available at the end of the article
}

important biological issue of wood, Yanase et al. $[4,5]$ introduced a gas analyzer equipped with a packed column and a semiconductor gas sensor as a detector. They qualified and quantified hydrogen and methane emitted from termite activities. Airborne substances can be collected by air sampling. Air sampling can be performed remotely and nondestructively.

Machine olfaction or an electronic nose is an odor classification method that combines multiple gas sensors and multivariate analysis. This method mimics mammal olfactory function by substituting olfactory receptor cells and the olfactory cortex with electronic sensors and a computer, respectively. The receptor cells of mammals have a small range of variation, and each variant responds to a broad range of substances. A particular type of odor is discriminated by a network of cross-connected receptor cells and neurons that process patterns of signals from variant receptors. In machine olfaction, odor discrimination is conducted by applying a pattern recognition algorithm to the response of multiple gas
Springer Open
C The Author(s) 2021. Open Access This article is licensed under a Creative Commons Attribution 4.0 International License, which permits use, sharing, adaptation, distribution and reproduction in any medium or format, as long as you give appropriate credit to the original author(s) and the source, provide a link to the Creative Commons licence, and indicate if changes were made. The images or other third party material in this article are included in the article's Creative Commons licence, unless indicated otherwise in a credit line to the material. If material is not included in the article's Creative Commons licence and your intended use is not permitted by statutory regulation or exceeds the permitted use, you will need to obtain permission directly from the copyright holder. To view a copy of this licence, visit http://creativecommons.org/licenses/by/4.0/. 
sensors [6]. Semiconductor gas sensors for specific gases have a high sensitivity to their target gases, but they also have a broad sensitivity to untargeted substances [7]. The sensor responses to individual odors vary among sensors depending on their gas-specific design. Because of this, a group of different gas-specific sensors, called a gas sensor array, generates unique patterns of output, often referred to as odor fingerprints for different odors [8]. The output of machine olfaction devices is represented by a vector with the dimension of the number of sensors. Principal component analysis (PCA) is commonly used to extract odor features. The PCA is also used to reduce data dimensions for data visualization. Supervised machine learning, for example, linear discriminant analysis or artificial neural network, is also applied for automated odor classification [9]. The advantages of machine olfaction over analytical methods are continuous monitoring [8], ease of building, cost-effectiveness, and short analysis time [9].

This method has been widely studied in food sciences, as odor is one of the most important factors of food. Machine olfaction is applied to evaluate food odor to avoid time-consuming analytical methods or sensory tests that are associated with unavoidable olfactory fatigue problems. Hui et al. [10] measured apple aroma and effectively predicted the storage time. Wei et al. [11] measured the odor of peanuts using machine olfaction and the acid and peroxide values of peanut kernels using traditional methods. The results of both analyses are highly correlated. Haugen et al. [12] measured the odor of spoilage in bacteria-inoculated milk using both machine olfaction and gas chromatography-mass spectrometry and revealed that both results were highly correlated. They concluded that machine olfaction could be used to detect milk spoilage.

In the field of wood sciences, Garneau et al. [13] demonstrated that some commercial electronic nose systems could classify three different softwood chips: spruce, fir, and pine. Baietto et al. [14] introduced several commercially available electronic noses for wood decay detection. They measured the odor of artificially infected wood samples and concluded that the electronic noses effectively detected wood decay. They used 23 fungal species and 18 wood species. They also measured the odor of inoculated tree root samples and concluded that the electronic nose could further detect root decay of trees [15]. Their scope was to investigate the decay of urban shade trees to prevent sudden collapse. Their choice of fungal cultures did not include common species found in wooden buildings such as T. versicolor, and most of the wood samples were hardwood. They used general-purpose laboratory-grade electronic noses. Furthermore, some of their data analysis depended on machine-specific built-in software.
Our final goal is to develop a wood constructionspecific on-site measuring device equipped with an automatic diagnosis algorithm using recent computing technology. As the first attempt to achieve the goal, we have developed a simple and cost-effective semiconductor gas sensor array that comprised commercially available components. Using the gas sensor array, we implemented a simple static headspace machine olfaction chamber. In this study, we aimed to examine the ability of a simple implementation of a machine olfaction device to detect odors from wood-decay fungi grown on common construction softwood. As representative wood-decay fungi, we selected $F$. palustris (brown-rot) and T. versicolor (white-rot). These species were chosen considering the availability of strains, information on cultivating conditions, and reported cases in wooden constructions [16], and they are also standard species in Japan Industrial Standard (JIS) [17]. As a typical construction softwood species, we selected sugi (Cryptomeria japonica) and karamatsu (Larix kaempferi). Both species are widely planted in Japan and commonly employed for construction. Sugi is also the standard species for JIS test methods for wood preservatives [17].

\section{Materials and methods}

\section{Fungal strains}

Two species were used in this study, namely $F$. palustris (Berkeley. et M.A. Curtis) Gilbertson et Ryvarden (FFPRI 0507) and T. versicolor (Linnaeus) Pilát (FFPRI 1030), hereinafter referred to as FP and TV, respectively. Both are standard species in JIS K 1571, the test methods for wood preservatives [17].

\section{Wood test pieces}

Test pieces were prepared following the sample preparation procedure of JIS K 1571 [17]. The samples were cut into test pieces with dimensions of $20 \mathrm{~mm} \times 20 \mathrm{~mm}$ in the axial section, and $10 \mathrm{~mm}$ in the fiber direction. The wood test pieces were kept for $48 \mathrm{~h}$ in a drying oven at $60 \pm 2{ }^{\circ} \mathrm{C}$, and then cooled for $30 \mathrm{~min}$ in a glass desiccator. After cooling, the initial weights of the test pieces were obtained. The test pieces were sterilized using ethylene oxide (EO) gas for $5 \mathrm{~h}$ at $40{ }^{\circ} \mathrm{C}$. We randomly picked six sterilized test pieces from each species as a sound-dry class. The test pieces were sealed in an air-tight plastic bag and maintained at $25 \pm 2{ }^{\circ} \mathrm{C}$.

\section{Cultivation of fungi on test pieces}

Three hundred and fifty grams of sea sand (Miyazaki Chemical, Tokyo, Japan), $100 \mathrm{~mL}$ of liquid medium $(\mathrm{d}-(+)$-glucose $4.0 \%(\mathrm{w} / \mathrm{w})$, peptone $0.3 \%(\mathrm{w} / \mathrm{w})$, and malt extract $1.5 \%(\mathrm{w} / \mathrm{w}))$ were added into each glass jar and autoclaved the jars at $60 \pm 2{ }^{\circ} \mathrm{C}$ for $30 \mathrm{~min}$. Then, mycelia 


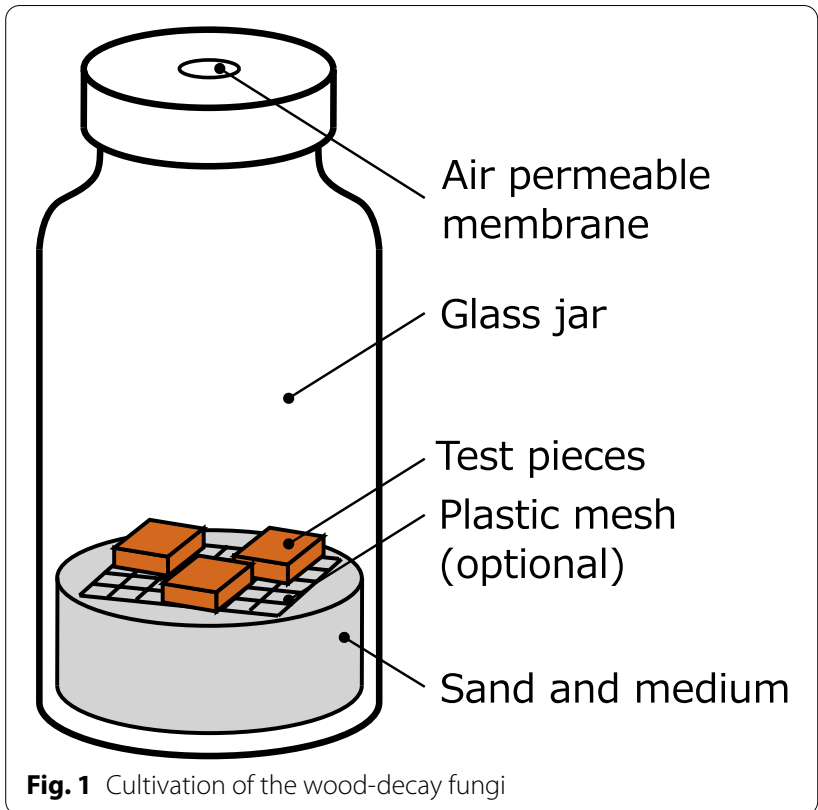

growing in a Petri dish on potato dextrose agar were inoculated at the medium center. The jars were maintained at $26{ }^{\circ} \mathrm{C}$ for 14 days. Thereafter, three pieces of wood were placed on the medium in each jar (Fig. 1). For FP, a plastic mesh sterilized by EO gas was placed between the test pieces and the medium. For TV, the test pieces were set directly on the medium. The jars were maintained at 26 $\pm 2{ }^{\circ} \mathrm{C}$ for $4,8,12$, and 20 weeks. These samples were named the decayed class. Two jars (six test pieces) were prepared for each condition. Non-inoculated jars were also prepared. The jars were processed using the same procedure described above except for inoculation and placed under the same conditions and periods. These samples were named the sound-wet class. This class was used to evaluate the effects of a medium odor.

\section{Equipment}

We developed a simple static headspace machine olfaction device equipped with a semiconductor gas sensor array. An array of six $\mathrm{SnO}_{2}$-based semiconductor gas sensors and a circulation fan was placed under the lid of a 7-L stainless steel chamber. A data logger (GL840, Graphtec, Yokohama, Japan) and a DC power supply were connected to the measuring chamber (Fig. 2). All gas sensors were commercially available devices for environmental monitoring, for example, leak detection and air purifiers (Table 1). Before measurement, we connected a clean air supplier (AOE2300, GL Sciences, Tokyo, Japan) to the chamber and aged the sensors in a clean air atmosphere at a flow rate of $100 \mathrm{~mL} / \mathrm{min}$ for 2 weeks.
Table 1 List of gas sensors

\begin{tabular}{llll}
\hline No. & Name & Target gases & Manufacturer \\
\hline 1 & TGS2600 & Hydrogen, ethanol & Figaro Eng. Inc. \\
2 & TGS2602 & Ammonia, $\mathrm{H}_{2} \mathrm{~S}$ & Figaro Eng. Inc. \\
3 & TGS2603 & Trimethylamine & Figaro Eng. Inc. \\
4 & TGS2620 & Alcohol, solvent vapors & Figaro Eng. Inc. \\
5 & TGS826 & Ammonia & Figaro Eng. Inc. \\
6 & MQ7 & Carbon monoxide & Henan Han- \\
& & & wei Electronics
\end{tabular}

\section{Measurement}

Before measurement, a $380-\mathrm{mL}$ stainless container filled with activated charcoal for an odorless air supplier (1050-3300, GL Sciences, Tokyo, Japan) was placed in the measuring chamber. The data logger was then set to record the output voltage of the gas sensors every $30 \mathrm{~s}$. After at least $12 \mathrm{~h}$, the sensor baseline values of a day were obtained. This sequence was performed every day during the measurement. A pair of test pieces was taken from the glass jars and placed on a glass Petri dish. Mycelia on the surface of the test pieces were kept as they were. The test pieces were quickly exchanged with the charcoal container in the measuring chamber. The chamber was then closed tightly. The voltage changes of the sensors $35 \mathrm{~min}$ after the chamber closure were recorded. After the measurement, the lid and test pieces were removed from the chamber. The chamber bucket was rinsed with distilled water, wiped with a paper towel, and then blown with hot air to remove moisture and odors. The charcoal container was placed again in the chamber. After $30 \mathrm{~min}$, the next measurement was performed. The measured test pieces were dried at room temperature for $24 \mathrm{~h}$, in a drying oven at $60 \pm 2{ }^{\circ} \mathrm{C}$ for $48 \mathrm{~h}$, and then cooled for $30 \mathrm{~min}$ in a glass desiccator. After cooling, the weights of the test pieces were obtained.

\section{Data processing}

The conductance of sensors was calculated from the sensor output voltage as follows:

$$
G_{i}=\frac{1}{R_{\mathrm{L}}\left(\frac{V_{\text {in }}}{V_{i}}-1\right)},
$$

where $G_{i}$ is the conductance (the reciprocal of the resistance) of sensor $i\left(\Omega^{-1}\right), R_{\mathrm{L}}$ is the resistance of the load resistor inserted in series to the sensors $(1 \mathrm{k} \Omega), V_{\mathrm{in}}$ is the voltage of the power supply $(\mathrm{V})$, and $V_{i}$ is the output 


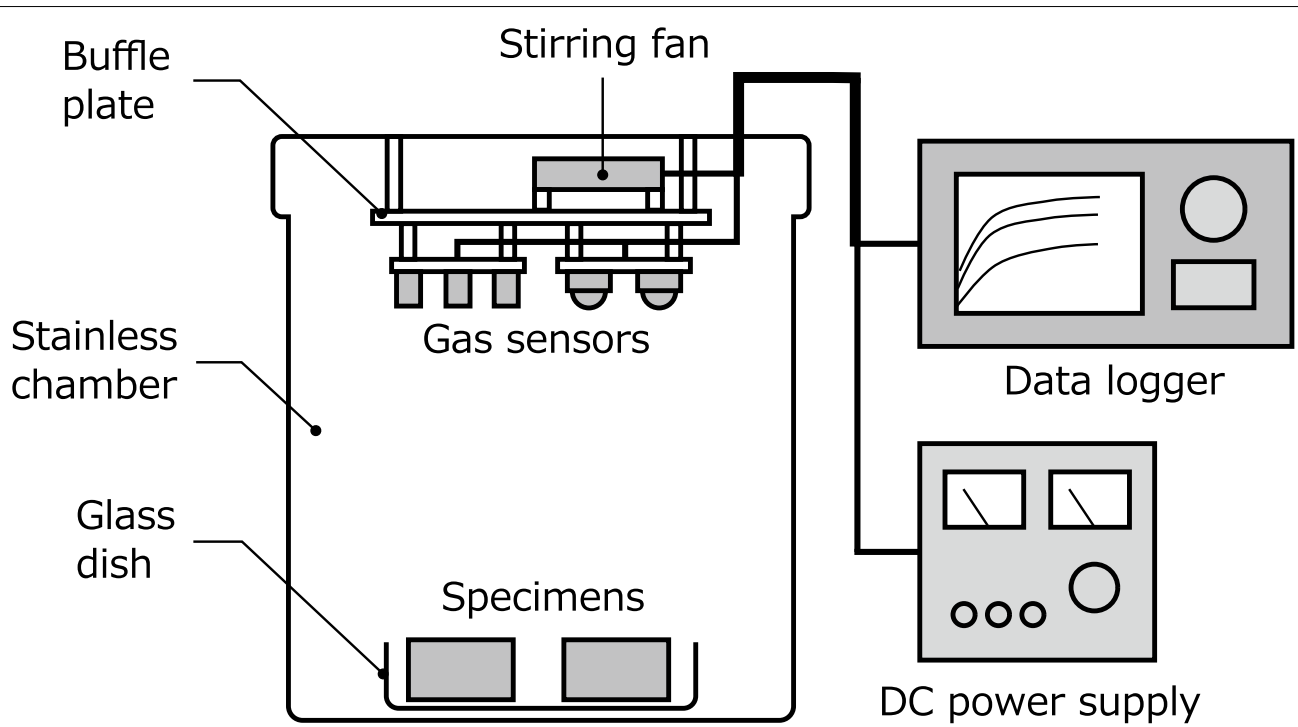

Fig. 2 Static headspace odor measurement device

voltage of sensor $i(\mathrm{~V})$. The sensor response was calculated as follows [18]:

$$
S_{i}=\frac{G_{i}-G_{0 i}}{G_{0 i}},
$$

where $S_{i}$ is the sensor response and $G_{0 i}$ is the baseline conductance of sensor $i\left(\Omega^{-1}\right)$.

The sensor responses were normalized to extract the features of the odor and to remove the concentrations of the vapors (odor strength) as follows [18]:

$$
\tilde{S}_{i}=\frac{S_{i}}{\sqrt{\sum_{i=1}^{n} S_{i}^{2}}},
$$

where $\tilde{S}_{i}$ is the normalized sensor response of sensor $i$.

PCA was performed on the normalized sensor responses using $\mathrm{R}$ 4.0.3 [19].

\section{Results and discussion}

Figure 3 shows the rate of mass loss of wood test pieces. FP showed a rapid mass loss for the first 12 weeks, which was followed by a slower loss in both wood species. TV showed a linear mass loss through the cultivation time in both wood species. Figure 4 shows representative raw sensor output voltages responding to the test pieces of FP on sugi at 20 weeks. The sensor output voltages showed a quick increase (conductance decrease) after the placement of the test pieces into the chambers (at $5 \mathrm{~min}$ ). The slope of the curves then decreased with time. The sensors used in the measurement showed sensitivity to the odors of the specimens. We used the values at $30 \mathrm{~min}$ after

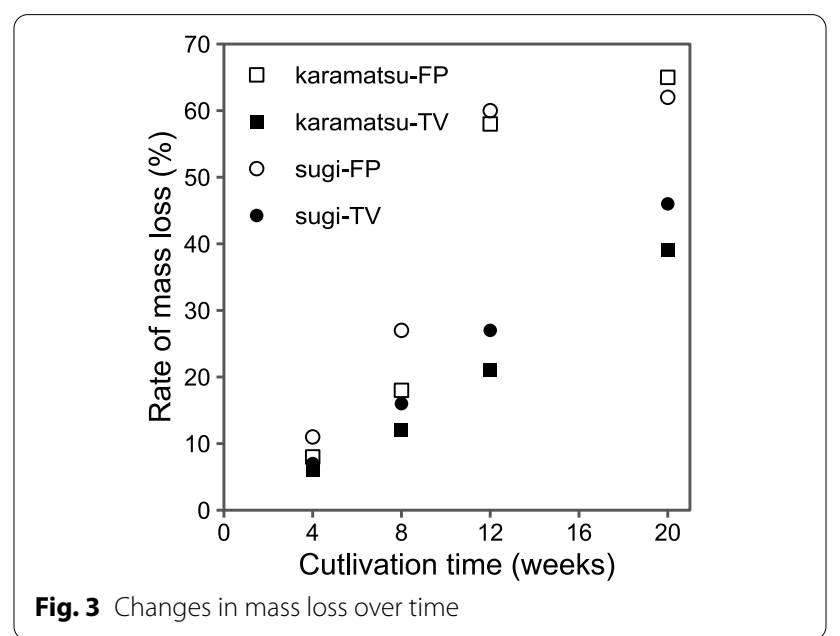

placing the test pieces based on the behavior of the sensor output. Figure 5 shows the normalized sensor response patterns of FP on sugi at 20 weeks. The normalized sensor responses showed unique patterns among the classes of specimens. The sound-dry class showed a distinctive pattern compared with the others. This difference can be explained by the low water content of the wood test pieces and the absence of a culture medium. Although fungi cultivation was applied only to the decayed class, both decayed class and sound-wet class showed similar patterns. This similarity could be because the sensor response was affected by the odor of the cultivation medium or wood test pieces with a higher moisture content. On the contrary, some sensors, especially sensor 


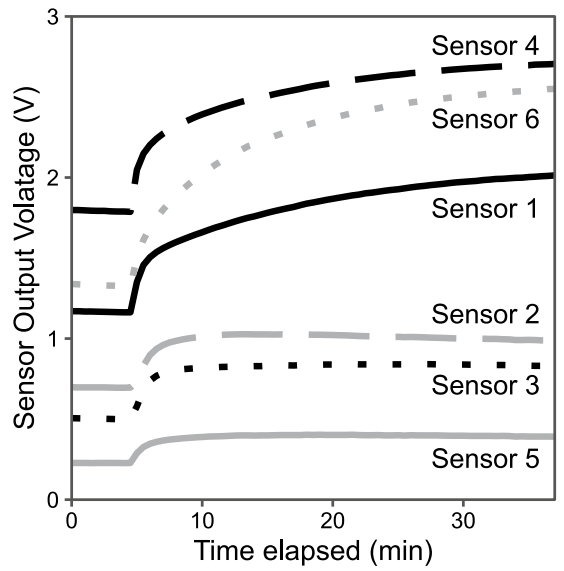

Fig. 4 Voltage change of the sensors responding to the decayed sample

3, showed different responses to these classes. These responses are considered to be fungi-related signals. To investigate whether the gas sensors could discriminate the fungi-related odor, the PCA was performed to reduce the data dimensions. Figure 6 shows scatter plots of principal components 1 and 2 of all measurements. In most of the measurements, each class formed an independent group in the plots except for FP karamatsu 8 weeks, TV sugi 4 weeks, and TV sugi 8 weeks. Overlapping distributions were found between decayed and sound-wet classes. The lower mass loss in the early stage of the cultivation might explain these overlaps. However, the relationship is unclear because it depends on both wood and fungal species. The decayed and sound-wet classes were closely distributed in the plots. This phenomenon shows that the sensors used in this study interpreted the odor of decayed and sound-wet classes as a similar odor. This result also reflects the patterns shown in Fig. 5. However, in most cases, the decayed class was separated from both sound-dry and sound-wet classes.

This result suggests that the odor of wood-decay fungi can be distinguished from that of sound wood using a gas sensor array. It could be presumed that decay detection using the gas sensor array was performed using a combination of MVOCs from wood-decay fungi and odor from sound wood. This hypothesis is based on a previous emission analysis. Konuma et al. [2] reported that wood-decay fungi emit various MVOCs, for example, 3-octanone. Suzuki [20] investigated three Japanese softwood species and reported that terpenes, including $\alpha$-pinene, were the predominant volatile organic compounds. In addition to the substances mentioned above, the odor of the culture medium and its change over time should not be ignored in experiments using a cultivation medium. The sensor responses to these odors could be an unintentional signal that could lead to an overestimation of the discrimination ability. Baietto et al. [14] reported a clearer discrimination in PCA plots than our results using commercial electronic noses. However, some parts of their preparation procedure for control (not inoculated) samples, for instance, the presence of medium or storage conditions, were unclear. In this study, we prepared sound-wet and sound-dry classes to clarify the effects of the treatment of sound samples. The sound-wet class was carefully prepared in the same way as the decayed class, except for mycelia inoculation. The sound-wet class appeared near the decayed class in the PCA plot and was also separated from the sound-dry class. This distribution can also be interpreted as the odor of the cultivation medium

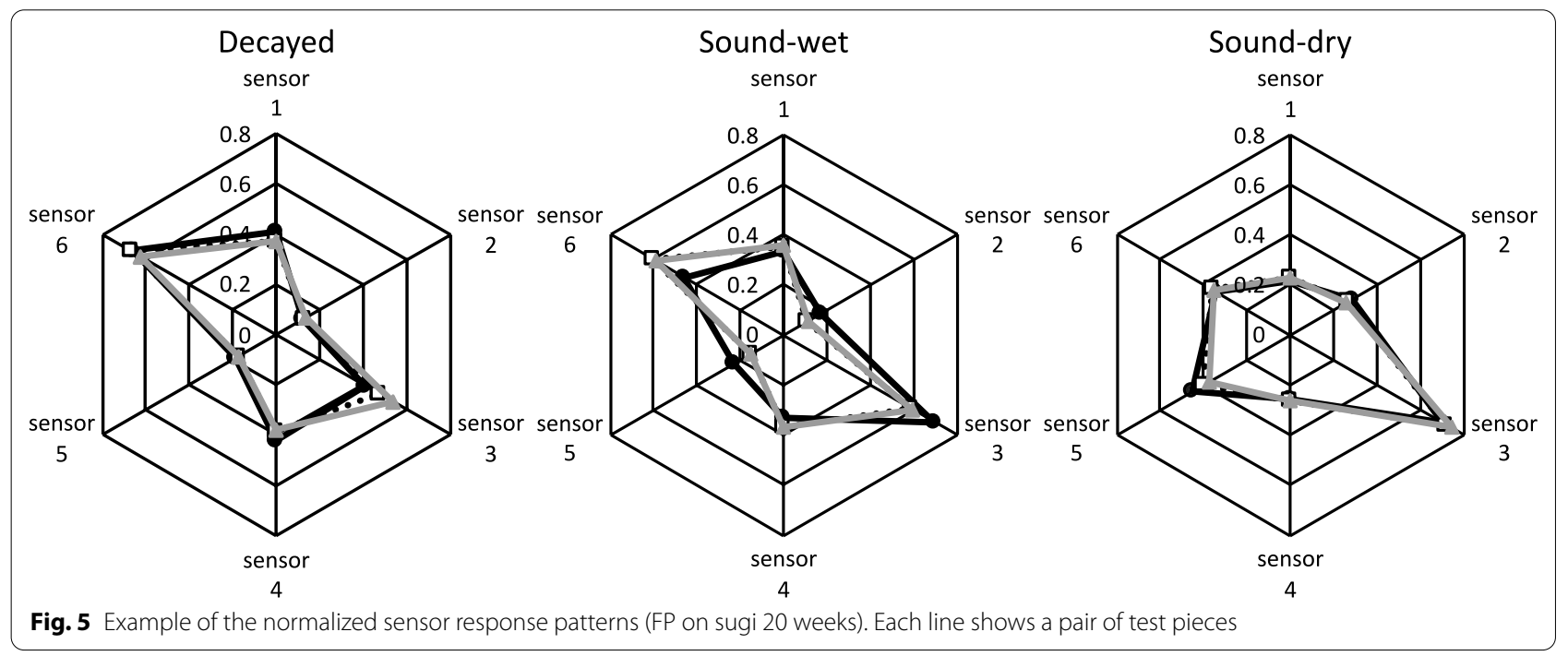




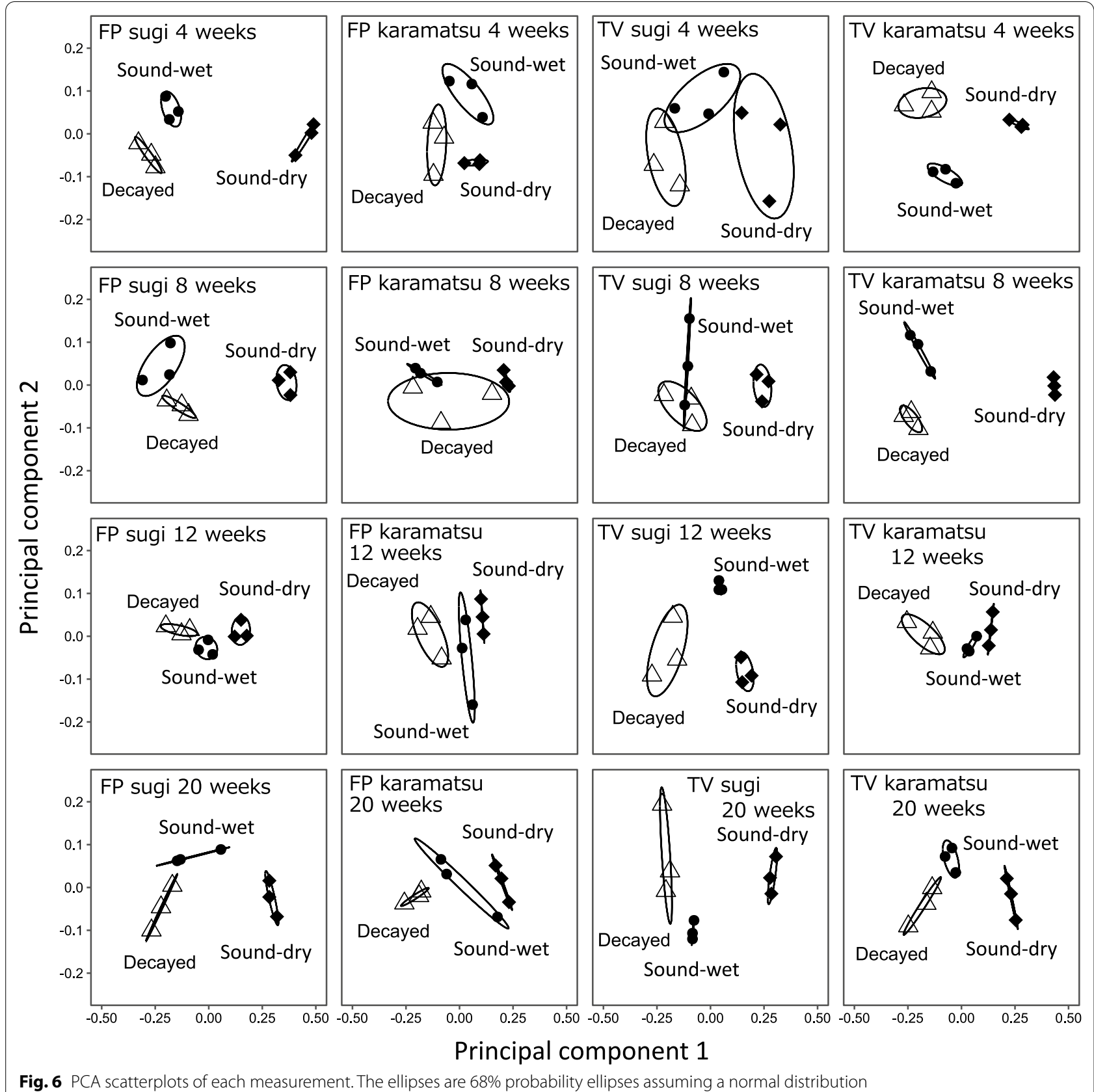

Fig. 6 PCA scatterplots of each measurement. The ellipses are 68\% probability ellipses assuming a normal distribution

transferred to the wood pieces, which is a mutually predominant odor in both decayed and sound-wet classes. If the control samples were inappropriately prepared, for example, a lack of cultivation medium or shortage of storage time, the gas sensor array would have detected the odor of the cultivation medium or its change over time. This would have been incorrectly interpreted as a signal of wood-decay fungi. Our results showed reasonable discrimination between the decayed and the two sound classes. Not only fungal MVOCs or terpenes, but the absence of the cultivation medium odor could also separate the sound-dry class and decayed class. On the contrary, the distinction between the sound-wet and decayed classes was also achieved in most cases. This suggests that the gas sensors responded to the fungirelated odor under the mutual cultivation medium odor.

To determine whether this method can distinguish wood-decay fungi-related odor from sound wood odor, the data of the measurement period and wood-decay fungal species were merged. In these new datasets, the 

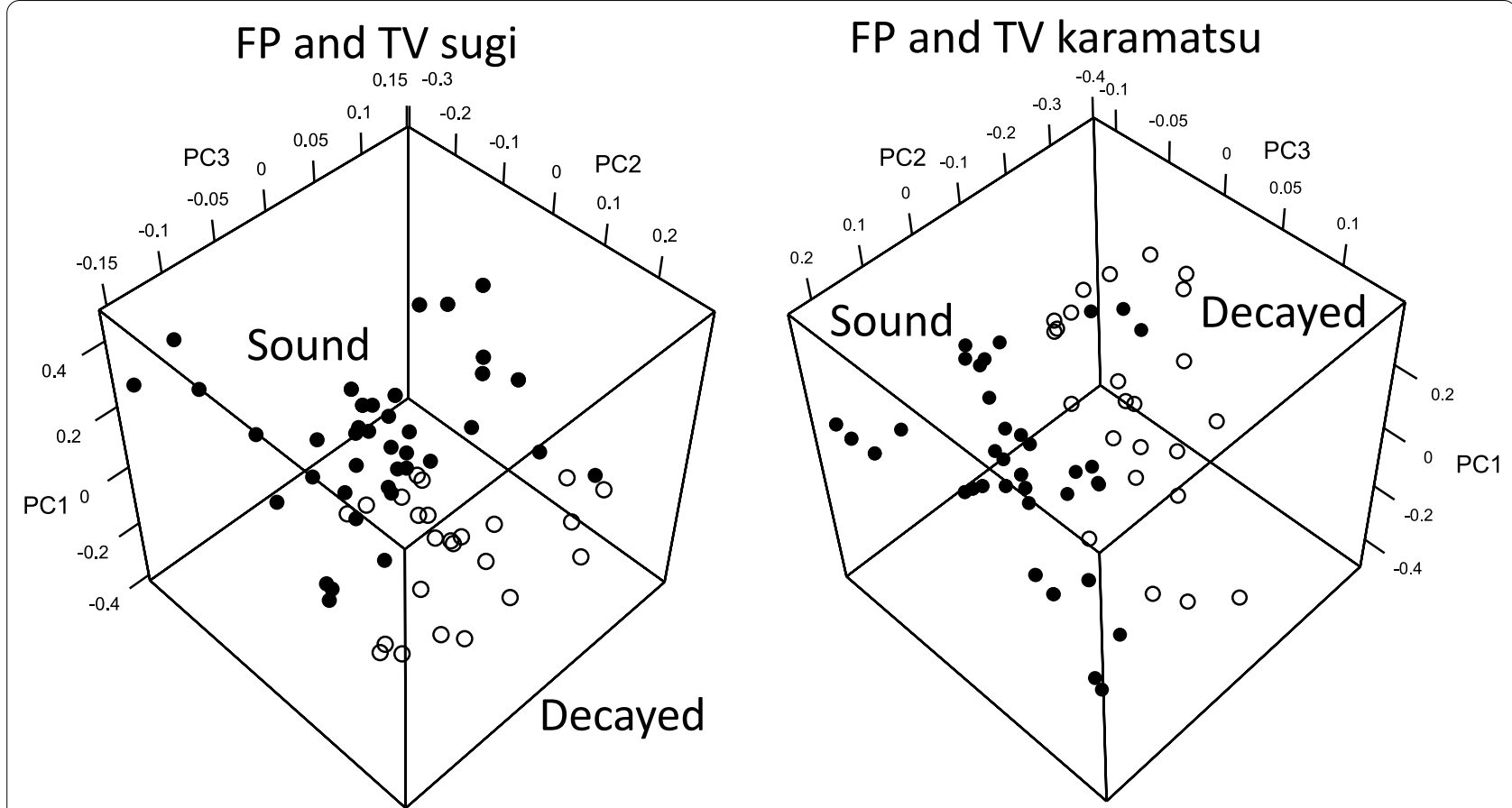

Fig. 7 PCA scatterplots of each wood species. PC: Principal component

sound-dry and sound-wet classes were also merged into a new sound class to simplify the problem, because these classes were not inoculated. Figure 7 shows scatter plots of principal components 1,2 , and 3 of the merged datasets. In both wood species, the decayed and sound classes formed individual groups in the plots. However, these groups partially overlapped with each other. These overlaps were comparatively small. The results also suggested that the gas sensor array detected specific patterns of the samples with wood-decay fungi and the sound samples, regardless of the species of wood-decay fungi and the cultivation period.

The simple device that we implemented is effective in detecting fungus-related odors in the combination of the two constructional softwoods and the two wooddecay fungi mentioned above. However, the present study has some limitations. It was conducted under pure cultivation conditions using limited fungal and wood species. Further, the sample sizes were small. Additionally, the static head space method that we used in this study required destructive sampling. Therefore, further research is needed under realistic cultivation conditions using non-destructive methods.

\section{Conclusions}

The findings of this study suggest that the simple semiconductor gas sensor array implemented in this study can detect the difference in odor between wood-decay fungi-inoculated wood and sound wood samples. The measured data of odor from inoculated samples and sound samples were reasonably well discriminated by PCA, regardless of the fungal species, wood species, or incubation period. In a pure culture experiment, the cultivation medium-related odor was predominant. The results suggest that our gas sensor array detected fungirelated odor from the samples prepared under pure culture conditions. Sensor-based odor discrimination enabled odor-based wood-decay fungi detection using a combination of JIS standard [17] fungal species and common construction wood. The approach evaluated in this study could be used as a cost-effective and easyto-use wood decay diagnosis. Furthermore, odor-based fungi or decay detection can be used to perform remote diagnoses when combined with air sampling methods.

\section{Abbreviations}

FP: Fomitopsis palustris; TV: Trametes versicolor; JIS: Japan Industrial Standards; EO: Ethylene oxide; PCA: Principal component analysis; MVOC: Microbial volatile organic compound.

\section{Acknowledgements}

We would like to thank Editage (www.editage.com) for English language editing. 


\section{Authors' contributions}

MS conceived the research concept, implemented the experimental device, performed measurements, analyzed data, and drafted the manuscript. TM designed the experiments and contributed to the fungal cultivation. SI contributed to fungal growth. YH contributed to the sample measurements. RN contributed to the design of the experimental devices and data analysis. All authors read and approved the final manuscript.

\section{Funding}

This work was supported by HRO ordinary research (Grant No. 3305-8W2901) and JSPS KAKENHI (Grant No. 19K06176).

\section{Availability of data and materials}

The datasets used and analyzed during this study are available from the corresponding author upon reasonable request.

\section{Declarations}

\section{Competing interests}

The authors declare that they have no competing interests.

\section{Author details}

${ }^{1}$ Forest Products Research Institute, Hokkaido Research Organization, 1-10 Nishikagura, Asahikawa, Hokkaido 071-0198, Japan. ${ }^{2}$ National Institute of Advanced Industrial Science and Technology (AIST), 16-1 Onogawa, Tsukuba, Ibaraki 305-8569, Japan.

Received: 11 July 2021 Accepted: 19 October 2021

Published online: 30 October 2021

\section{References}

1. Kauhanen E, Harri M, Nevalainen A, Nevalainen T (2002) Validity of detection of microbial growth in buildings by trained dogs. Environ Int 28(3):153-157. https://doi.org/10.1016/S0160-4120(02)00021-1

2. Konuma R, Umezawa K, Mizukoshi A, Kawarada K, Yoshida M (2015) Analysis of microbial volatile organic compounds produced by wooddecay fungi. Biotechnol Lett 37(9):1845-1852. https://doi.org/10.1007/ s10529-015-1870-9

3. Konuma R, Mizukoshi A, Kawarada K, Yoshida M (2015) Analysis of microbial volatile organic compounds emitted by brown rot fungus Fomitopsis palustris. Mokuzai Hozon (Wood Protection) 41(3):108-118. https://doi. org/10.5990/jwpa.41.108 (in Japanese with English summary)

4. Yanase Y, Miura M, Fujii Y, Okumura S, Yoshimura T (2013) Evaluation of the concentrations of hydrogen and methane emitted by termite using a semiconductor gas sensor. J Wood Sci 59(3):243-248. https://doi.org/10. 1007/s10086-013-1325-7

5. Yanase Y, Maruyama S, Fujii Y, Okumura S, Yoshimura T (2013) Detection of hydrogen and methane emitted by feeding activity of termite under forced ventilation. Japn J Environ Entomol Zool 24(3):97-105. https://doi. org/10.11257/jjeez.24.97

6. Persaud K, Dodd G (1982) Analysis of discrimination mechanisms in the mammalian olfactory system using a model nose. Nature 299:352-355
7. Gardner JW, Bartlett PN (1994) A brief history of electronic noses. Sens Actuat B Chemical 18(1):210-211. https://doi.org/10.1016/0925-4005(94) 87085-3

8. Patel HK (2013) Toward Sensor to Reproduce Human Sense. In: The Electronic Nose: Artificial Olfaction Technology. Biological and Medical Physics, Biomedical Engineering, Springer, New Delhi, pp 115-142, https://doi. org/10.1007/978-81-322-1548-6

9. Peris M, Escuder-Gilabert $L$ (2009) A 21st century technique for food control: Electronic noses. Analytica Chimica Acta 638(1):1-15. https://doi. org/10.1016/j.aca.2009.02.009

10. Guohua H, Yuling W, Dandan Y, Wenwen D (2013) Fuji Apple storage time predictive method using electronic nose. Food Anal Methods 6(1):82-88. https://doi.org/10.1007/s12161-012-9414-6

11. Wei Z, Wang J, Zhang W (2015) Detecting internal quality of peanuts during storage using electronic nose responses combined with physicochemical methods. Food Chem 177:89-96. https://doi.org/10.1016/j. foodchem.2014.12.100

12. Haugen JE, Rudi K, Langsrud S, Bredholt S (2006) Application of gassensor array technology for detection and monitoring of growth of spoilage bacteria in milk: A model study. Analytica Chimica Acta 565(1):10-16. https://doi.org/10.1016/j.aca.2006.02.016

13. Garneau FX, Riedl B, Hobbs S, Pichette A, Gagnon H (2004) The use of sensor array technology for rapid differentiation of the sapwood and heartwood of Eastern Canadian spruce, fir and pine. Holz als Roh Werkstoff 62(6):470-473. https://doi.org/10.1007/s00107-004-0508-8

14. Baietto M, Wilson AD, Bassi D, Ferrini F (2010) Evaluation of three electronic noses for detecting incipient wood decay. Sensors 10(2):10621092. https://doi.org/10.3390/s100201062

15. Baietto M, Pozzi L, Wilson AD, Bassi D (2013) Evaluation of a portable MOS electronic nose to detect root rots in shade tree species. Computers Electron Agric 96:117-125. https://doi.org/10.1016/j.compag.2013.05.002

16. Sakai H, Kakutani T, Tezuka D, Shigeyama T, Sugai Y, Shintani T, Yamaguchi A (2020) Decay resistance of wood and treated wood to 12 strains of fungi. Mokuzai Hozon (Wood Protection) 46(4):196-201. https://doi.org/ 10.5990/jwpa.46.196 (in Japanese with English summary)

17. Japanese Standards Association (2010) Wood preservatives - performance requirements and their test methods for determining effectiveness. Standard JIS K 1571:2001, Japanese Standards Association, Tokyo, Japan (in Japanese)

18. Gardner J, Hines E, Tang H (1992) Detection of vapours and odours from a multisensor array using pattern-recognition techniques part 2. artificial neural networks. Sens Actuat Chem 9(1):9-15. https://doi.org/10.1016/ 0925-4005(92)80187-3

19. R Core Team (2020) R: A Language and Environment for Statistical Computing. R Foundation for Statistical Computing, Vienna, Austria, https:// www.R-project.org/

20. Suzuki M (2019) Bayesian modeling of volatile organic compound emissions from three softwoods in Hokkaido. J Wood Sci, Japan. https://doi. org/10.1186/s10086-019-1790-8

\section{Publisher's Note}

Springer Nature remains neutral with regard to jurisdictional claims in published maps and institutional affiliations. 